\title{
Clinicopathological implications of leptin and leptin receptor expression in papillary thyroid cancer
}

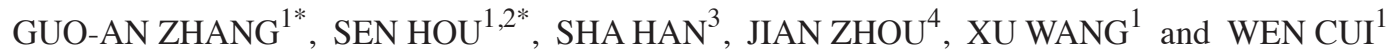 \\ ${ }^{1}$ Department of Pathology, Jining Medical University, Jining, Shandong 272067; \\ ${ }^{2}$ Institute of Basic Medicine, Shandong Institute of Medicine, Jinan, Shandong 250001; ${ }^{3}$ Life Science Experimental Center; \\ ${ }^{4}$ Center of Forensic Science, Jining Medical University, Jining, Shandong 272067, P.R. China
}

Received September 30, 2012; Accepted December 18, 2012

DOI: $10.3892 / \mathrm{ol} .2013 .1125$

\begin{abstract}
The role of leptin and its receptors (OBRs) in the pathogenesis of various primary human malignancies has been demonstrated. However, their expression and clinicopathological significance in papillary thyroid cancer (PTC) is not fully understood. In this study, we examined the expression of leptin and OBRs in 76 PTC samples using immunohistochemistry, and their associations with clinicopathological parameters were evaluated. The expression of OBRs was observed in the tumor cell membrane and/or cytoplasm, with a positive rate of $73.7 \%$ (56/76), while leptin was expressed in the tumor cell cytoplasm in 55 of 76 cases $(72.4 \%)$. The expression of either protein was associated with greater tumor size $(\mathrm{P}=0.016$ for leptin and $\mathrm{P}=0.002$ for $\mathrm{OBRs}$ ). In addition, the expression levels of leptin and OBRs were associated with each other. Neither leptin nor OBR expression levels were associated with other parameters, including age, body weight, postmenopausal state, multifocality and lymph node metastasis. These data suggest that the expression of leptin and/or OBRs in PTC is associated with tumor size and may be a potential target in PTC.
\end{abstract}

\section{Introduction}

Thyroid cancer is the most common malignancy of the endocrine system and accounts for $\sim 1 \%$ of all newly diagnosed cancer cases in the USA $(1,2)$. The most frequent type is papillary thyroid cancer (PTC), which constitutes $>80 \%$ of all cases. It has also been reported that the thyroid cancer incidence rate has significantly increased among males and females in numerous countries, including the USA (1), Southeast

Correspondence to: Dr Wen Cui, Department of Pathology, Jining Medical University, 16 Beihu Road, Jining, Shandong 272067, P.R. China

E-mail: cuiwenmd@yahoo.com

\section{${ }^{*}$ Contributed equally}

Key words: leptin, leptin receptors, clinicopathological features, papillary thyroid cancer
England (3), Italy (4), Lithuania (5), Canada (6) and China (7), and enhanced medical scrutiny of small tumors cannot explain this finding $(1,2)$.

Obesity is now becoming an epidemic worldwide (8), including in China $(9,10)$. It has been considered as a risk factor for several types of cancer, including colorectal cancer, postmenopausal breast cancer, kidney cancer and endometrial cancer (11). For thyroid cancer, a meta-analysis (12) found that a $5-\mathrm{kg} / \mathrm{m}^{2}$ increase in body mass index (BMI) was strongly associated with thyroid cancer in males $(1.33, \mathrm{P}=0.02)$ and females $(1.14, \mathrm{P}=0.001)$, although the association is weaker in females.

One of the important mediators between obesity and increased cancer risk is leptin $(11,13)$, which is an adipokine whose major functions are regulating appetite and energy homeostasis (14). Leptin serum levels are closely correlated with adiposity in humans. It exerts its effects through binding to its receptors (OBRs), which are located in several tissues throughout the body. Among the receptors, only the long form $(\mathrm{OBRb})$ was considered to have full potential to transduce signals. The signaling pathways activated by OBRb include the classic cytokine Janus kinase 2/signal transducer and activator of transcription 3 (JAK2/STAT3) pathway; the Ras/extracellular signal-regulated kinases 1/2 (Ras/ERK1/2) signaling cascade; and the phosphoinositide 3 kinase/protein kinase B (PI3K/Akt) growth/anti-apoptotic pathway (15).

Leptin and OBRs have been reported to be overexpressed in numerous types of cancer and cancer cell lines (16). Moreover, their expression intensity is associated with cancer progression and/or prognosis in several common types of cancer, including glioblastoma (17), breast cancer (18), prostate cancer (19), ovarian cancer $(20)$ and colorectal cancer $(21,22)$, as revealed by immunohistochemical studies.

Leptin and OBR expression has also been studied in thyroid cancers. In a study on a large cohort of Saudi PTC patients (23), OBRs and leptin were found to be expressed in $80.1 \%(410 / 512)$ and $49.1 \%$ (252/513) of PTC patients, respectively, and OBR expression was strongly associated with age, gender, extrathyroidal extension, tumor stage, tumor size, node metastasis and histological type. However, in another study of Chinese PTC patients in Taiwan (24), different results were obtained. OBRs and leptin were detected in $51.0 \%$ (25 of 49) and $36.7 \%$ (18 of 49) of cases, respectively, and neither 
were associated with age, gender, extrathyroidal extension, multifocality, thyroiditis or BMI. The exception was tumor size, which was shown to be associated with OBR and leptin expression. The study also revealed that PTC tumors with both leptin and OBR expression were more likely to develop lymph node metastasis compared with tumors with neither leptin nor OBR expression. However, reasons for the differences between the results of the two studies are unknown.

The aim of the present study was to detect the expression of leptin and OBRs in a group of Chinese mainland PTC patients, and to determine whether their expression correlated with patient and tumor characteristics.

\section{Patients and methods}

Patients. This study included 76 patients who underwent thyroidectomy in Jining First People's Hospital between 2010 and 2011. Clinical and histopathological data, including tumor size, multifocality, lymph node metastasis, age and weight, were reviewed for all patients. Hematoxylin and eosin (HE)-stained slides for each patient were reviewed to confirm the diagnosis of PTC. All patients were euthyroid prior to surgery. The ethics committee of the Affiliated Hospital of Jining Medical College censored and approved the study. Written informed consent was obtained from the patients.

Immunohistochemistry (IHC). For immunohistochemical staining, 5-mm sections of formalin-fixed, paraffin-embedded tissue blocks were dewaxed in xylene and rehydrated through graded alcohol and phosphate-buffered saline (PBS). Antigen retrieval was conducted by boiling slides in $10 \mathrm{mM}$ sodium citrate buffer ( $\mathrm{pH} \mathrm{6.0)}$ for $10 \mathrm{~min}$. The sections were then treated with $0.3 \%$ hydrogen peroxide at room temperature for $10 \mathrm{~min}$ to quench endogenous peroxidase activity. After rinsing in PBS, the sections were blocked with $10 \%$ normal rabbit serum (for OBRs) or goat serum (for leptin) at $37^{\circ} \mathrm{C}$ for $1 \mathrm{~h}$. Then the sections were incubated overnight at $4^{\circ} \mathrm{C}$ in humid chambers with primary antibody. The primary polyclonal rabbit anti-leptin antibody (A-20, Santa Cruz Biotechnology, Inc., Santa Cruz, CA, USA) was diluted at 1:200 and the primary polyclonal goat anti-OBR antibody (M-18, for all forms of OBRs; Santa Cruz Biotechnology, Inc.) was diluted at 1:200. Subsequently, the sections were incubated with a polyperoxidase-conjugated anti-rabbit or anti-goat secondary antibody (Gold Bridge, Beijing, China) for $30 \mathrm{~min}$. Following a PBS wash, DAB substrate (Gold Bridge) was added to the sections for $30 \mathrm{~min}$. Finally, the slides were counterstained with hematoxylin, dehydrated after a standard procedure and sealed with coverslips. Sections that had been incubated with PBS instead of primary antibody were used as a negative control.

Evaluation of immunostaining. The expression levels of leptin and OBRs were evaluated semiquantitatively by two experienced pathologists (X.W. andW.C.). Scoring was based on staining intensity and staining extent. Staining intensity was scored as 0 (negative), 1 (weak), 2 (moderate) or 3 (strong). Staining extent was scored as $0(0 \%), 1(1-25 \%), 2(26-50 \%)$ or $3(51-100 \%)$ according to the percentage of positively stained cells. Multiplied scores of intensity and extent were used as the
Table I. Correlation between OBR and leptin expression.

\begin{tabular}{lcrrr}
\hline & \multicolumn{2}{c}{ Leptin expression } & & \\
\cline { 2 - 3 } $\begin{array}{l}\text { OBR } \\
\text { expression }\end{array}$ & Positive & Negative & Total & P-value \\
\hline Positive & 44 & 12 & 56 & \\
Negative & 11 & 9 & 20 & \\
Total & 55 & 21 & 76 & $P<0.05$ \\
\hline
\end{tabular}

OBR, leptin receptor.

final staining score. Patients were sorted into 2 groups; positive expression was defined by final staining scores of 6 and 9 , whereas the remaining cases (final scores $0-4$ ) were classified as negative expression.

Statistical analysis. The correlation between the expression of OBRs/leptin and clinicopathological features was analyzed. Continuous variables are expressed as the mean \pm standard deviation (SD). Data were evaluated for significant differences by the 2-tailed Student's t-test or the $\chi^{2}$ test using the Statistical Package for Social Sciences, version 13.0 (SPSS, Inc., Chicago, IL, USA). P $<0.05$ was considered to indicate statistically significant differences.

\section{Results}

Immunohistochemical detection of OBRs and leptin. Expression levels of OBRs and leptin were determined by IHC in 76 PTC samples. OBR expression was observed in tumor cell membrane and/or cytoplasm with a positive rate of $73.7 \%$ (56/76), while leptin was expressed in tumor cell cytoplasm in 55 of 76 cases (72.4\%; Fig. 1). In 57.9\% (44/76) of cases, both leptin and OBRs were expressed. The expression levels of OBRs and leptin in PTC samples were significantly associated with each other, as shown in Table I $(\mathrm{P}<0.05)$.

Association of expression of leptin and OBRs with clinicopathological parameters. Our study on the association of OBR and leptin expression with clinicopathological data demonstrated that PTC samples with positive staining for OBRs $(\mathrm{P}=0.002)$ or leptin $(\mathrm{P}=0.016)$ were associated with a larger tumor size. Neither leptin nor OBR expression was associated with other parameters, including age, body weight, postmenopausal state, multifocality or lymph node metastasis (Table II).

\section{Discussion}

Our study showed that $73.9 \%(56 / 76)$ and $72.4 \%(55 / 76)$ of PTC samples expressed OBRs and leptin, respectively. These data are different from those of Cheng et al (24) who revealed that OBRs and leptin are expressed in 51.0\% (25/49) and 36.7\% (18/49) of PTC cases, respectively, and Uddin et al (23) whose results showed that OBRs and leptin are expressed in $80.1 \%$ (410/512) and $49.1 \%$ (252/513) of PTC cases, respectively. Although the positive rates are different, all three studies indicated that a proportion of PTC tumors express leptin and 
Table II. Expression of leptin and OBRs relative to the clinicopathological characteristics of PTC.

\begin{tabular}{|c|c|c|c|c|c|c|}
\hline \multirow[b]{2}{*}{ Characteristic } & \multicolumn{3}{|c|}{ Leptin expression } & \multicolumn{3}{|c|}{ OBR expression } \\
\hline & Positive & Negative & P-value & Positive & Negative & P-value \\
\hline No. of patients & 55 & 21 & & 56 & 20 & \\
\hline Age (years), mean \pm SD & $46.0 \pm 13.3$ & $53.4 \pm 12.3$ & 0.878 & $47.7 \pm 14.7$ & $48.9 \pm 8.9$ & 0.736 \\
\hline Body weight $(\mathrm{kg})$, mean $\pm \mathrm{SD}$ & $66.5 \pm 10.8$ & $68.4 \pm 11.9$ & 0.427 & $67.2 \pm 12$ & $66.2 \pm 8.5$ & 0.703 \\
\hline $\begin{array}{l}\text { No. of postmenopausal patients } \\
\text { (56 female patients) }(\%)\end{array}$ & $14(33.3)$ & $6(42.9)$ & 0.520 & $15(35.7)$ & $5(35.7)$ & 1.000 \\
\hline Lymph node metastasis (\%) & $17(30.9)$ & $9(42.9)$ & 0.326 & $20(35.7)$ & $6(30)$ & 0.644 \\
\hline Tumor size $(\mathrm{mm})$, mean $\pm \mathrm{SD}$ & $22.2 \pm 10.3$ & $17.1 \pm 12.8$ & 0.016 & $23.7 \pm 10.1$ & $15.1 \pm 10.3$ & 0.002 \\
\hline Multifocality $(\%)$ & $31(56.4)$ & $14(66.7)$ & 0.414 & $32(57.1)$ & $13(65.0)$ & 0.539 \\
\hline
\end{tabular}

OBR, leptin receptor; PTC, papillary thyroid cancer; SD, standard deviation.
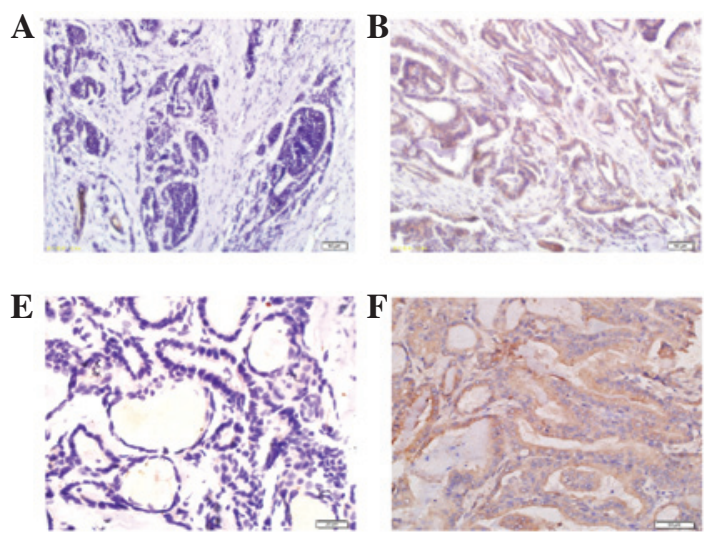
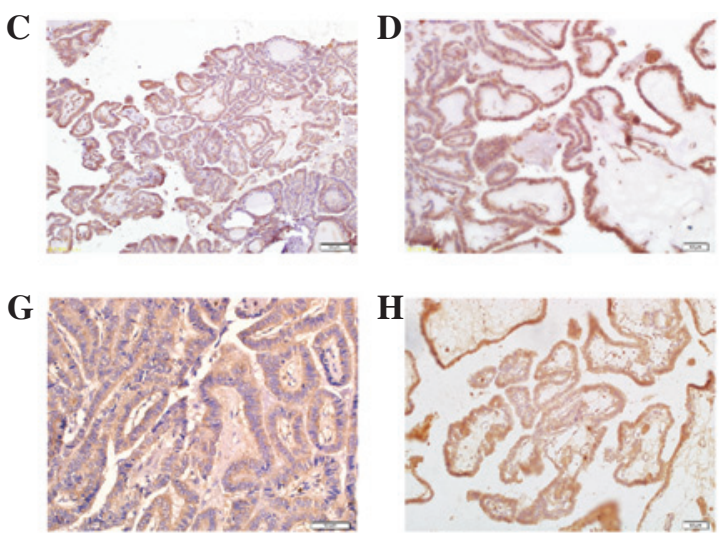

Figure 1. Leptin and OBR expression in PTC samples. (A) Negative, (B) weak, (C) moderate and (D) strong expression of leptin in PTC samples is shown in the first row. The second row demonstrates expression of OBRs with (E) negative, $(\mathrm{F})$ weak, $(\mathrm{G})$ moderate and (H) strong staining. OBR, leptin receptor; PTC, papillary thyroid cancer.

OBRs. However, the reasons for differences between the data requires further study, particularly the difference between the data of this study and those of Cheng et al, due to the similar ethnicity of the PTC patients and also since the same antibodies were used.

In this study, $57.9 \%$ of PTC cases expressed both leptin and OBRs, and it was also observed that the expression levels of leptin and OBRs were significantly associated with each other. Similar results were obtained by Cheng et al (24) in PTC and in other cancer types, including endometrial cancer (25), colorectal cancer $(26)$ and breast cancer $(18,27)$. This suggests that leptin and OBR expression may be induced by some of the same mechanisms, or that the expression of one molecule may be induced by the other. Indeed, leptin and OBR expression is correlated with HIF-1 $\alpha$ in endometrial (25) and colorectal cancer (26), which suggests that HIF-1 $\alpha$ may induce both. More direct evidence from in vitro cell line studies indicated that IGF-1, insulin and estradiol induced the expression of leptin and OBR mRNA in the MCF-7 breast cancer cell line, while in MDA-MB-231 cells, leptin and OBR mRNA expression was induced by insulin or hypoxia (28). There is also evidence demonstrating that leptin enhanced the expression of OBRs, for example in ZR-75-1 breast cancer cells (29). Whether or not these mechanisms exist in PTC cells is largely unknown. However, insulin was previously shown to upregulate OBR expression in a time- and dose-dependent manner, while the hypoxia-mimicking agent cobalt chloride had no effect on OBR expression in PTC cell lines (30). The effect of insulin on leptin expression in thyroid cancer has not been studied.

In the present study, leptin and OBR expression levels were found to be associated with PTC tumor size, which is similar to the observations of two previous studies $(23,24)$. This result is to be expected, considering that leptin, through OBRs, has been shown to promote proliferation and inhibit apoptosis in numerous types of cancer $(16)$ and PTC cell lines $(23,31,32)$.

We have identified several weaknesses in this study. Firstly, the small number of patients enrolled means there is a higher chance of producing imprecise results, compared with a larger group of patients. The second and more important point is the lack of study of the survival rate or recurrence in these patients due to the relatively benign nature of PTC. However, Uddin et al (23) showed that patients with overexpression of OBRs had a reduced disease-free survival rate of $68.9 \%$ at 5 years, compared with $79.3 \%$ with reduced OBR expression. 
In summary, our observations have added to the evidence that leptin and OBRs are expressed in PTC and their expression is associated with each other and with PTC tumor size. Further study is required to determine the potential prognostic and therapeutic implications of the leptin/OBR system.

\section{Acknowledgements}

This study was supported by the Natural Science Foundation of Shandong province (ZR2009CM070), Jining Scientific and Technological Project (2011-31) and Youth Foundation of Jining Medical University (JYQ2011KM006).

\section{References}

1. Simard EP, Ward EM, Siegel R and Jemal A: Cancers with increasing incidence trends in the United States: 1999 through 2008. CA Cancer J Clin: Jan 4, 2012 (Epub ahead of print).

2. Chen AY, Jemal A and Ward EM: Increasing incidence of differentiated thyroid cancer in the United States, 1988-2005. Cancer 115: 3801-3807, 2009.

3. Olaleye O, Ekrikpo U, Moorthy R, et al: Increasing incidence of differentiated thyroid cancer in South East England: 1987-2006. Eur Arch Otorhinolaryngol 268: 899-906, 2011.

4. Capezzone M, Morabito E, Bellitti P, Giannasio P, De Sanctis D and Bruno R: Increasing incidence of thyroid cancer in Basilicata: an Italian study. J Endocrinol Invest 30: 507-512, 2007.

5. Smailyte G, Miseikyte-Kaubriene E and Kurtinaitis J: Increasing thyroid cancer incidence in Lithuania in 1978-2003. BMC Cancer 6: 284,2006

6. Liu S, Semenciw R, Ugnat AM and Mao Y: Increasing thyroid cancer incidence in Canada, 1970-1996: time trends and age-period-cohort effects. Br J Cancer 85: 1335-1339, 2001.

7. Xiang J, Wu Y, Li DS, et al: New clinical features of thyroid cancer in eastern China. J Visc Surg 147: e53-e56, 2010.

8. Finucane MM, Stevens GA, Cowan MJ, et al: National, regional, and global trends in body-mass index since 1980: systematic analysis of health examination surveys and epidemiological studies with 960 country-years and 9.1 million participants. Lancet 377: 557-567, 2011.

9. Wang Y, Mi J, Shan XY, Wang QJ and Ge KY: Is China facing an obesity epidemic and the consequences? The trends in obesity and chronic disease in China. Int J Obes (Lond) 31: 177-188, 2007.

10. Chen CM: Overview of obesity in Mainland China. Obes Rev 9 (Suppl 1): 14-21, 2008.

11. Khandekar MJ, Cohen P and Spiegelman BM: Molecular mechanisms of cancer development in obesity. Nat Rev Cancer 11: 886-895, 2011.

12. Renehan AG, Tyson M, Egger M, Heller RF and Zwahlen M: Body-mass index and incidence of cancer: a systematic review and meta-analysis of prospective observational studies. Lancet 371: 569-578, 2008.

13. Roberts DL, Dive C and Renehan AG: Biological mechanisms linking obesity and cancer risk: new perspectives. Annu Rev Med 61: 301-316, 2010.
14. Zhang Y, Proenca R, Maffei M, Barone M, Leopold L and Friedman JM: Positional cloning of the mouse obese gene and its human homologue. Nature 372: 425-432, 1994.

15. Sweeney G: Leptin signalling. Cell Signal 14: 655-663, 2002.

16. Garofalo C and Surmacz E: Leptin and cancer. J Cell Physiol 207: 12-22, 2006.

17. Riolfi M, Ferla R, Del Valle L, et al: Leptin and its receptor are overexpressed in brain tumors and correlate with the degree of malignancy. Brain Pathol 20: 481-489, 2010.

18. Ishikawa M, Kitayama J and Nagawa H: Enhanced expression of leptin and leptin receptor (OB-R) in human breast cancer. Clin Cancer Res 10: 4325-4331, 2004.

19. Hoon Kim J, Lee SY, Myung SC, Kim YS, Kim TH and Kim MK: Clinical significance of the leptin and leptin receptor expressions in prostate tissues. Asian J Androl 10: 923-928, 2008.

20. Uddin S, Bu R, Ahmed M, et al: Overexpression of leptin receptor predicts an unfavorable outcome in Middle Eastern ovarian cancer. Mol Cancer 8: 74, 2009.

21. Koda M, Sulkowska M, Kanczuga-Koda L, Surmacz E and Sulkowski S: Overexpression of the obesity hormone leptin in human colorectal cancer. J Clin Pathol 60: 902-906, 2007.

22. Liu H, Wan D, Pan Z, et al: Expression and biological significance of leptin, leptin receptor, VEGF, and CD34 in colorectal carcinoma. Cell Biochem Biophys 60: 241-244, 2011.

23. Uddin S, Bavi P, Siraj AK, et al: Leptin-R and its association with PI3K/AKT signaling pathway in papillary thyroid carcinoma. Endocr Relat Cancer 17: 191-202, 2010.

24. Cheng SP, Chi CW, Tzen CY, et al: Clinicopathologic significance of leptin and leptin receptor expressions in papillary thyroid carcinoma. Surgery 147: 847-853, 2010.

25. Koda M, Sulkowska M, Wincewicz A, et al: Expression of leptin, leptin receptor, and hypoxia-inducible factor 1 alpha in human endometrial cancer. Ann NY Acad Sci 1095: 90-98, 2007.

26. Koda M, Sulkowska M, Kanczuga-Koda L, et al: Expression of the obesity hormone leptin and its receptor correlates with hypoxia-inducible factor-1 alpha in human colorectal cancer. Ann Oncol 18 (Suppl 6): vil16-119, 2007.

27. Koda M, Kanczuga-Koda L, Sulkowska M, Surmacz E and Sulkowski S: Relationships between hypoxia markers and the leptin system, estrogen receptors in human primary and metastatic breast cancer: effects of preoperative chemotherapy. BMC Cancer 10: 320, 2010.

28. Garofalo C, Koda M, Cascio S, et al: Increased expression of leptin and the leptin receptor as a marker of breast cancer progression: possible role of obesity-related stimuli. Clin Cancer Res 12: 1447-1453, 2006.

29. Chen C, Chang YC, Liu CL, Chang KJ and Guo IC: Leptininduced growth of human ZR-75-1 breast cancer cells is associated with up-regulation of cyclin D1 and c-Myc and downregulation of tumor suppressor p53 and p21WAF1/CIP1. Breast Cancer Res Treat 98: 121-132, 2006.

30. Cheng SP, Liu CL, Hsu YC, Chang YC, Huang SY and Lee JJ: Regulation of leptin receptor expression in human papillary thyroid cancer cells. Biomed Pharmacother 66: 469-473, 2012.

31. Cheng SP, Yin PH, Chang YC, Lee CH, Huang SY and Chi CW: Differential roles of leptin in regulating cell migration in thyroid cancer cells. Oncol Rep 23: 1721-1727, 2010.

32. Cheng SP, Yin PH, Hsu YC, et al: Leptin enhances migration of human papillary thyroid cancer cells through the PI3K/AKT and MEK/ERK signaling pathways. Oncol Rep 26: 1265-1271, 2011. 